\title{
Relations Between Resistance Distances of a Graph and its Complement or its Contraction ${ }^{\dagger}$
}

\author{
Yujun Yang, ${ }^{\mathrm{a}, \mathrm{b}, \mathrm{c}}$ \\ ${ }^{a}$ School of Mathematics and Information Science, Yantai University, Yantai, Shandong, 264005, P.R. China. \\ ${ }^{\mathrm{b}}$ Mathematical chemistry group, Texas A\&M University at Galveston, Galveston, Texas, 77553-1675, USA \\ 'School of Mathematics, Shandong University, Jinan, Shandong, 250100, P.R. China \\ (E-mail:yangy@tamug.edu,yangyj@yahoo.com)
}

RECEIVED JUNE 13, 2013; REVISED JANUARY 1, 2014; ACCEPTED JANUARY 3, 2014

\begin{abstract}
The resistance distance between two vertices of a connected graph is defined as the net effective resistance between them when each edge of the graph is replaced by a resistor. In this paper, it is shown that the product of resistance distances between any pair of vertices in a simple graph and in its connected complement is less than or equal to 3. Meanwhile, a relation between resistance distances of a graph and its contraction is obtained in a special case. (doi: $10.5562 / \mathrm{cca} 2318$ )
\end{abstract}

Keywords: resistance distance, graph complement, graph contraction, Rayleigh's short-cut principle

\section{INTRODUCTION}

Two decades ago, a novel distance function on graphs was identified by Klein and Randić. ${ }^{1}$ They viewed a connected graph as an electrical network by imagining that fixed resistors are assigned to each edge. Then they proved that the effective resistance between pairs of vertices is a distance function on the graph and named this new distance function resistance distance.

As a central component of electric circuit theory, effective resistance have long been studied in physics and engineering, dating back to Kirchhoff ${ }^{2}$ and Maxwell, ${ }^{3}$ and extending on to modern electrical engineering, see, e.g., Seshu \& Reed, ${ }^{4}$ and Chan. ${ }^{5}$ Besides, it has been shown that effective resistance is also relevant to a wide range of problems ranging from random walks on graphs, ${ }^{6}$ the theory of harmonic functions ${ }^{7}$ to lattice Green's functions. ${ }^{8}$ However, not until the contribution of Klein and Randić, ${ }^{1}$ it is revealed that the effective resistance is an intrinsic graph metric. Thanks to this important fact, people came to realize that resistance distance is a fundamental concept of graph theory and began to consider its mathematical properties as well as its applications in chemistry. Since then, resistance distance has aroused the great interest of mathematicians and chemists, especially chemical graph theorists.

The utilization of resistance distance in chemistry (and even mathematics) is still mostly potential. While various invariants (such as the analogue to the Wiener index-the Kirchhoff index, ${ }^{1}$ the degree Kirchhoff index ${ }^{9}$ and the additive degree Kirchhoff index ${ }^{10}$ ) may be used as another one of the many topological indices to make QSPRs or QSARs, ${ }^{11-18}$ we believe that the resistance distance has a more fundamental role. Being a natural intrinsic metric for graphs, which indeed is motivatable in different ways, ${ }^{9,19-23}$ such as then indicates is diverse relevance. Notably resistance distance can be seen to be useful in quantifying cyclicity, ${ }^{24,25}$ or centrality ${ }^{26}$ of a graphical structure-each idea of which is of fundamental interest in chemistry, and much beyond. This contrasts with what is done with almost all topological indices which are introduced to distinguish (nonisomorphic) graphs or to explore possible correlations with different properties. Thus development of the basic understandings of resistance distance is worthy activity, to try to understand it better, and thence its possible chemical applications. These different motivations are by way of different interpretations: combinatorially, as a normalized number of spanning bi-trees each tree containing one of the indexed pair of sites; ${ }^{4}$ as a waveamplitude correlation function; ${ }^{20}$ as an effective electrical resistance between pairs of sites, ${ }^{1}$ in terms of a random walk probability of going from one site to another without returning to the first ${ }^{6}$ etc. That each of these different motivations leads to the same metric, marks the consequent metric as fundamental, and likely to be the proper distance to use in a variety of different contexts, including chemical.

\footnotetext{
$\dagger$ Dedicated to Professor Douglas Jay Klein on the occasion of his $70^{\text {th }}$ birthday.
} 
For more information on resistance distance, the readers are referred to Refs. 27-46.

\section{RELATIONS BETWEEN RESISTANCE DIS- TANCES OF A GRAPH AND ITS COMPLEMENT}

In this section, we only consider simple graphs, that is, every edge of the graph is assigned a unit resistor. Let $G=(V(G), E(G))$ be a connected graph. For any $i, j \in V(G)$, we use $\Omega_{G}(i, j)$ and $d_{G}(i, j)$ to denote the resistance distance and the (shortest-path) distance between $i$ and $j$, respectively. For $X \subseteq V(G)$, the subgraph induced by $X$, denoted by $G[X]$, is defined as the graph whose vertex set is $X$ and whose edge set consists of all the edges of $G$ with both end vertices in $X$. The complement of $G$, denoted by $\bar{G}$, is a graph on the same vertices such that two vertices of $\bar{G}$ are adjacent if and only if they are not adjacent in $G$. In Ref. 47, Deng and Chen considered the Kirchhoff index of a bipartite graph and its complement. Here we consider resistance distance of a graph and its complement. In this section, an upper bound for the resistance distance between any two vertices in $\bar{G}$ is given in terms of (shortest path) distances between them in $G$, which leads to an upper bound for the product of the resistance distance between any two vertices in $G$ and the resistance distance between them in $\bar{G}$.

We begin with the famous Rayleigh's short-cut principle, which is fundamental in electrical network theory. Here cutting involves nothing more than clipping some of the edges of the network, or what is the same, simply deleting them from the network. Shorting involves connecting a given set of vertices together with perfectly conducting wires, so that current can pass between them with resistance zero. The principle is stated as follows:

Proposition 2.1. (Rayleigh's short-cut principle) ${ }^{6}$ Shorting certain sets of vertices together can only decrease the effective resistance of the network between two given vertices. Cutting certain edges can only increase the effective resistance between two given vertices.

We also need the formula for computing resistance distances of circulant graphs. Recall that a circulant graph is a graph whose adjacency matrix is a circulant matrix. A circulant matrix $C=\left(c_{i j}\right)_{n \times n}$ of order $n$ is a matrix such that the entries $c_{i j}$ satisfy $c_{i j}=c_{1, j-i+1}$ (the second subscript module $n$ ); that is, $C$ has the form:

$$
C=\left[\begin{array}{ccccc}
c_{0} & c_{1} & c_{2} & \cdots & c_{n-1} \\
c_{n-1} & c_{0} & c_{1} & \cdots & c_{n-2} \\
c_{n-2} & c_{n-1} & c_{0} & \cdots & c_{n-3} \\
\vdots & \vdots & \vdots & \ddots & \vdots \\
c_{1} & c_{2} & c_{3} & \cdots & c_{0}
\end{array}\right] .
$$

Noticing that a circulant graph could be uniquely determined by its first low, so the circulant matrix $C$ can also be expressed in terms of its first row as $C=C\left[c_{0}, c_{1}, c_{2}, \ldots, c_{n-1}\right]$.

For a circulant graph $G$, it is easily verified that the Laplacian matrix of $G$ is a circulant matrix too. Thus we may suppose that $L(G)=C\left[l_{0}, l_{1}, l_{2}, \cdots, l_{n-1}\right]$. Then resistance distances in $G$ can be computed as

Theorem 2.2. ${ }^{48}$ For $n$-vertex circulant graph $G(n \geq 2)$,

$$
\begin{aligned}
\Omega_{G}(i, j)= & \frac{4}{n} \sum_{k=1}^{n-1}\left(\sum_{s=0}^{n-1} l_{s} \cos \frac{2 k s \pi}{n}\right)^{-1} \sin ^{2} \frac{(i-j) k \pi}{n} \\
& {\left[1-\sin \frac{2(i+j-2) k \pi}{n}\right] . }
\end{aligned}
$$

Lemma 2.3. ${ }^{48}$ Suppose that $k, n$ are positive integers. Then the following identity holds:

$$
\sum_{s=1}^{n-1} \sin \frac{2 k s \pi}{n}=0 .
$$

If, in addition, $k<n$, we also have

$$
\sum_{s=0}^{n-1} \cos \frac{2 k s \pi}{n}=0 .
$$

Using Theorem 2.2 and Lemma 2.3, we have the following Lemma.

Lemma 2.4. Let $C_{n}$ be the cycle graph on $n$ vertices. For any two nonadjacent vertices $i$ and $j$ of $\overline{C_{n}}$ $(n \geq 5)$, we have

$$
\Omega_{\bar{C}_{n}}(i, j) \leq \frac{2}{n-4} .
$$

The proof of Lemma 2.4 is given in Appendix A.

As a byproduct of Lemma 2.4 , it turns out that the resistance distance between any two nonadjacent vertices in $\overline{C_{n}}$ could be in a rather narrow interval.

Corollary 2.5. For any two nonadjacent vertices $i$ and $j$ of $\overline{C_{n}}(n \geq 5)$,

$$
\frac{2}{n-3}<\Omega_{\overline{C_{n}}}(i, j) \leq \frac{2}{n-4} .
$$

Proof. It suffices to show the lower bound. As shown in Ref. 49, for any two nonadjacent vertices $u$ and $v$,

$$
\Omega_{G}(u, v) \geq \frac{1}{d_{G}(u)}+\frac{1}{d_{G}(v)},
$$

with equality if and only if $u$ and $v$ have the same neighbor set. Hence it follows directly that 


$$
\Omega_{\overline{C_{n}}}(i, j)>\frac{1}{n-3}+\frac{1}{n-3}=\frac{2}{n-3} .
$$

Using Lemma 2.4, we could give a lower bound for the resistance distance between any two vertices in $\bar{G}$ in terms of the distance between them in $G$.

Proposition 2.6. For any $i, j \in V(G)=V(\bar{G})$,

$$
\Omega_{\bar{G}}(i, j) \leq\left\{\begin{array}{cl}
1, & \text { if } d_{G}(i, j)=2 \text { or } 3 \\
\frac{2}{d_{G}(i, j)-1}, & \text { if } d_{G}(i, j) \geq 4
\end{array}\right.
$$

Proof. If $d_{G}(i, j)=2$ or 3 , then $i$ and $j$ are adjacent in $\bar{G}$. Thus it is straightforward to see that

$$
\Omega_{\bar{G}}(i, j) \leq d_{\bar{G}}(i, j)=1 .
$$

Now suppose that $d_{G}(i, j) \geq 4$. Choose a shortest path $P$ connecting $i$ and $j$ in $G$. One may see that for any two nonadjacent vertices in $P$, they must be not adjacent in $G$ and be adjacent in $\bar{G}$. It follows that the induced subgraph of $V(P)$ in $\bar{G}$ is $\bar{P}$, that is, $\bar{G}[V(P)]=\bar{P}$. Since $\bar{P}$ is a subgraph of $\bar{G}$, by Rayleigh's short-cut principle, we have

$$
\Omega_{\bar{G}}(i, j) \leq \Omega_{\bar{P}}(i, j) .
$$

To estimate $\Omega_{\bar{P}}(i, j)$, we construct a graph $\bar{C}$ from $\bar{P}$ by removing the edge $i j$. Observe that $\bar{C}$ is the complement of the cycle obtained from $P$ by connecting $i$ and $j$. Then by the well-known parallel connection rule, which states that resistors that are connected in parallel can be replaced by a single resistor whose conductance (the inverse of resistance) is the sum of conductances, we readily have

$$
\frac{1}{\Omega_{\bar{P}}(i, j)}=\frac{1}{\Omega_{\bar{C}}(i, j)}+\frac{1}{1} .
$$

Consequently,

$$
\Omega_{\bar{P}}(i, j)=\frac{\Omega_{\bar{C}}(i, j)}{\Omega_{\bar{C}}(i, j)+1} .
$$

Observe by Lemma 2.4 that

$$
\Omega_{\bar{C}}(i, j) \leq \frac{2}{|V(\bar{C})|-4}=\frac{2}{d_{G}(i, j)-3} .
$$

Hence
$\Omega_{\bar{P}}(i, j)=\frac{\Omega_{\bar{C}}(i, j)}{\Omega_{\bar{C}}(i, j)+1} \leq \frac{\frac{2}{d_{G}(i, j)-3}}{\frac{2}{d_{G}(i, j)-3}+1}=\frac{2}{d_{G}(i, j)-1}$

It follows immediately that

$$
\Omega_{\bar{G}}(i, j) \leq \Omega_{\bar{P}}(i, j) \leq \frac{2}{d_{G}(i, j)-1} .
$$

According to Proposition 2.6, we have the following proposition.

Proposition 2.7. Let $G$ be a connected graph with a connected $\bar{G}$. Then for any $i, j \in V(G)=V(\bar{G})$,

$$
\Omega_{G}(i, j) \cdot \Omega_{\bar{G}}(i, j) \leq 3
$$

Proof. If $d_{G}(i, j)=2$ or 3 , then $\Omega_{\bar{G}}(i, j) \leq 1$ and clearly the assertion holds.

$$
\begin{gathered}
\text { If } d_{G}(i, j)>3, \text { by Theorem 2.6, } \\
\Omega_{\bar{G}}(i, j) \leq \frac{2}{d_{G}(i, j)-1} . \text { Hence } \\
\Omega_{G}(i, j) \cdot \Omega_{\bar{G}}(i, j) \leq d_{G}(i, j) \cdot \Omega_{\bar{G}}(i, j) \\
\leq \frac{2 d_{G}(i, j)}{d_{G}(i, j)-1}=\frac{2}{1-\frac{1}{d_{G}(i, j)}} \leq \frac{2}{1-\frac{1}{4}}=\frac{8}{3}<3,
\end{gathered}
$$

as desired. If $d_{G}(i, j)=1$, then $d_{\bar{G}}(i, j) \geq 2$. By applying the above arguments to $\bar{G}$, we also have

$$
\Omega_{G}(i, j) \cdot \Omega_{\bar{G}}(i, j) \leq 3 .
$$

Note that the bound is sharp. In addition, from the proof of Proposition 2.7 we can see that a sharper upper bound can be obtained in terms of $d_{G}(i, j)$.

For example, we consider the path graph $P_{4}$ and the benzene graph $B$ (see Figure 1). Resistance distances in both of them and their complements are given in Table 1 and Table 2. Since the resistance distance between any two vertices in both $B$ and $\bar{B}$ depends

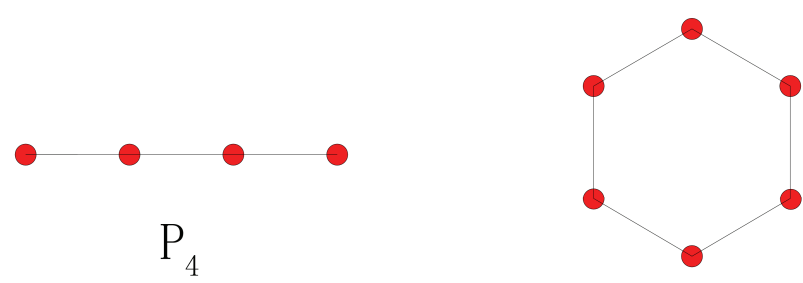

Figure 1. The path graph $P_{4}$ (left) and the benzene graph $B$ (right). 
Table 1. Resistance distances in $P_{4}$ and its complement

\begin{tabular}{cccc}
\hline$(i, j)$ & $\Omega_{p 4}(i, j)$ & $\Omega_{\overline{p 4}}(i, j)$ & $\Omega_{p 4}(i, j) \Omega_{\overline{p 4}}(i, j)$ \\
\hline$(1,2)$ & 1 & 2 & 2 \\
$(1,3)$ & 2 & 1 & 2 \\
$(1,4)$ & 3 & 1 & 3 \\
$(2,3)$ & 1 & 3 & 3 \\
$(2,4)$ & 2 & 1 & 2 \\
$(3,4)$ & 1 & 2 & 2 \\
\hline
\end{tabular}

Table 2. Resistance distances in the benzene graph and its complement

\begin{tabular}{cccc}
\hline$(i, j)$ & $\Omega_{B}(i, j)$ & $\Omega_{\bar{B}}(i, j)$ & $\Omega_{B}(i, j) \Omega_{\bar{B}}(i, j)$ \\
\hline$d_{B}(i, j)=1$ & $5 / 6$ & $5 / 11$ & $11 / 18$ \\
$d_{B}(i, j)=2$ & $4 / 3$ & $\frac{8}{15}+\frac{\sqrt{3}}{10}$ & $\frac{32}{45}+\frac{2 \sqrt{3}}{15}$ \\
$d_{B}(i, j)=3$ & $3 / 2$ & $3 / 5$ & $9 / 10$ \\
\hline
\end{tabular}

only on the distance between them in $B$, it suffices to distinguish resistance distances in $B$ and $\bar{B}$ into three classes.

\section{RELATION BETWEEN RESISTANCE DIS- TANCES OF A GRAPH AND ITS CONTRACTION}

In this section, we consider resistance distances in the graph obtained from a graph by contracting a set of vertices. Let $G$ be a connected simple graph and let $S$ be a proper subset of $V(G)$. We use $G-S$ to denote the graph obtained from $G$ by deleting all the vertices of $S$. We denote $G / S$ the graph obtained from $G$ by contracting $S$ into a single vertex, i.e., replacing $S$ with a single vertex and connect this single vertex to every neighbor of $S$. Notice that multiple edges may result after the contraction. To avoid multiple edges, by the parallel connection rule, if two vertices in $G / S$ are connected by $k$ edges, then we could replace these edges with a single edge of resistance $1 / k$. Hence edges in $G / S$ may not necessarily represent a unit resistor. To distinguish these graphs with (simple) graphs whose edges represent unit resistors, we call such graphs weighted graphs. Hence in a weighted graph, edges can represent resistors of arbitrary values. In the following, it is understood that $G$ is a simple graph and its contractions may be weighted graphs.

By Rayleigh's short-cut principle, it is straightforward to have the following property.
Proposition 3.1. Let $G$ be a connected graph. For $S \subset V(G)$, let $G / S$ be defined as above. Then for any two vertices $p, q \in V(G / S)$,

$$
\Omega_{G / S}(p, q) \leq \Omega_{G}(p, q) .
$$

In particular, if $|S|=2$, then as shown in Ref. 46 , resistance distances in $G / S$ could be computed in terms of resistance distances in $G$ as given in the following formula.

Theorem 3.2. (Ref. 46, Theorem 4.5) Let $G$ be a connected weighted graph and let $S=\{i, j\} \subset V(G)$. Then for any two vertices $p, q \in V(G / S)$,

$$
\begin{aligned}
& \Omega_{G / S}(p, q)=\Omega_{G}(p, q)- \\
& \frac{\left[\Omega_{G}(p, i)+\Omega_{G}(q, j)-\Omega_{G}(p, j)-\Omega_{G}(q, i)\right]^{2}}{4 \Omega_{G}(i, j)} .
\end{aligned}
$$

Noting that Theorem 3.2 is applicable to weighted graphs and using it repeatedly, we could get resistance distances in $G / S$ in terms of resistance distances in $G$. But the expression may become too complicated to write down. It motivates us to consider whether we could find a simpler expression for resistance distances in $G / S$ if $S$ is chosen to have some specified properties. Fortunately, if $S$ satisfies that all vertices in $S$ have the same neighborhood $N$ in $G-S$, then we obtain a very simple expression.

Proposition 3.3. Let $G$ be a connected (simple) graph. Let $S \in V(G)$ such that all vertices in $S$ have the same neighborhood $N$ in $G-S$ and $G[S]$ is an independent set. If $p, q \in V(G) \backslash S$, then

$$
\Omega_{G / S}(p, q)=\Omega_{G}(p, q)
$$

whereas if $p \in V(G) \backslash S$ and $q \in S$, then

$$
\Omega_{G / S}(p, q)=\Omega_{G}(p, q)-\frac{|S|-1}{|S||N|} .
$$

The proof of Proposition 3.3 could be found in Appendix B.

As an simple example, we use Proposition 3.3 to compute resistance distance in the complete bipartite graph $K_{m, n}$ with parts of sizes $m$ and $n$ (see Figure 2 (left)). Choose the $m$-sites part as $S$, then by contracting $S$ in $K_{m, n}$, we obtain the graph $K_{m, n} / S$ (see Figure 2 (right)). Then for any $a, b$ from the $n$-sites part and $x$ from the $m$-sites part, by Proposition 3.3, simple calculation could lead to 

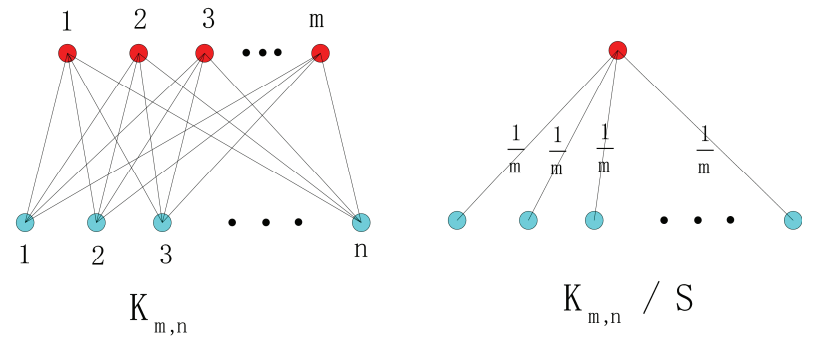

Figure 2. The complete bipartite graph $K_{m, n}$ (left) and the graph obtained from $K_{m, n}$ by contracting the $m$-sites part $S$ (right). Note that $K_{m, n} / S$ is a weighted graph and the numbers on edges of $K_{m, n} / S$ indicate weights (also resistances) on these edges.

$$
\Omega_{K_{m, n}}(a, b)=\frac{2}{m}, \quad \Omega_{K_{m, n}}(a, x)=\frac{m+n-1}{m n} .
$$

It coincides with the result obtained in Ref. 32.

\section{CONCLUDING REMARKS AND FUTURE WORKS}

Resistance distance is a very important and interesting graph metric with various applications in physics, engineering and chemistry. The main contribution of this paper is twofold. First, some intrinsic relations between resistance distances in a connected $G$ and its complement are established. To the best of my knowledge, it is the first time to discuss the relation between resistance distances in a graph and its complement although the discussion is still at primary stage. Second, resistance distances in $G / S$ are expressed in terms of resistance distances of $G$ provided that $S$ is chosen to satisfy that all vertices in $S$ have the same neighborhood $N$ in $G-S$. It promotes study on relations of resistance distances between a graph and its contraction.

Along the line of the present manuscript, there might be some problems worthy of study in the future. For example, what other relations do there exist between resistance distances in a graph and its complement? Could we find simple expressions for resistance distances in $G / S$ in terms of resistance distances of $G$ if $S$ is any subset of vertices of $G$ ?

Supplementary Materials. - Supporting informations to the paper are enclosed to the electronic version of the article. These data can be found on the website of Croatica Chemica Acta (http://public.carnet.hr/ccacaa).

Acknowledgements. The author would like to thank anonymous referees for their valuable comments and constructive suggestions, which greatly improve the manuscript. The support of the Welch Foundation of Houston, Texas (through grant BD-0894), National Natural Science Foundation of China (through grant 11201404), China Postdoctoral Science
Foundation (through grants 2012M521318 and 2013T60662), Special Funds for Postdoctoral Innovative Projects of Shandong Province (through grant 201203056), are greatly acknowledged.

\section{APPENDIX A: PROOF OF LEMMA 2.4.}

Proof. Suppose that the vertices $V\left(C_{n}\right)=V\left(\overline{C_{n}}\right)$ $=\{1,2, \ldots, n\}$ and vertices in $C_{n}$ are labeled in cyclic order. It is easily verified that for $n \geq 5, \overline{C_{n}}$ is a connected circulant graph with Laplacian matrix

$$
L\left(\overline{C_{n}}\right)=C[n-3,0, \overbrace{-1, \ldots,-1}^{n-3}, 0] .
$$

Hence by Theorem 2.2,

$$
\begin{aligned}
& \Omega_{\overline{C_{n}}}(i, j)= \\
& \frac{4}{n} \sum_{k=1}^{n-1}\left(n-3-\sum_{s=2}^{n-2} \cos \frac{2 k s \pi}{n}\right)^{-1} \sin ^{2} \frac{(i-j) k \pi}{n} \\
& {\left[1-\sin \frac{2(i+j-2) k \pi}{n}\right] .}
\end{aligned}
$$

Note that resistance distances between all pairs of nonadjacent vertices are equal in $\overline{C_{n}}$. Without loss of generality, it suffices to consider the resistance distance between vertices 1 and $n$ in $\overline{C_{n}}$. By Equation (5.1) and Lemma 2.3, we have

$$
\begin{aligned}
& \Omega_{\overline{C_{n}}}(i, j)=\Omega_{\overline{C_{n}}}(1, n) \\
&=\frac{4}{n} \sum_{k=1}^{n-1} \frac{\sin ^{2} \frac{(n-1) k \pi}{n}\left[1-\sin \frac{2(n-1) k \pi}{n}\right]}{n-\left(\sum_{s=0}^{n-1} \cos \frac{2 k s \pi}{n}-1-\cos \frac{2 k \pi}{n}-\cos \frac{2(n-1) k \pi}{n}\right)} \\
&=\frac{4}{n} \sum_{k=1}^{n-1}\left[n-3-\left(0-1-\cos \frac{2 k \pi}{n}-\cos \frac{2(n-1) k \pi}{n}\right)\right]^{-1} \\
& \sin ^{2} \frac{(n-1) k \pi}{n}\left[1-\sin \frac{2(n-1) k \pi}{n}\right] \\
&=\frac{4}{n} \sum_{k=1}^{n-1}\left(n-2+\cos \frac{2 k \pi}{n}+\cos \frac{2 k(n-1) \pi}{n}\right)^{-1} \sin ^{2} \frac{(n-1) k \pi}{n} \\
& {\left[1-\sin \frac{2(n-1) k \pi}{n}\right] } \\
& \leq \frac{4}{n} \sum_{k=1}^{d}(n-4)^{-1} \sin ^{2} \frac{(n-1) k \pi}{n}\left[1-\sin \frac{2(n-1) k \pi}{n}\right] \\
&= \frac{4}{n(n-4)} \sum_{k=1}^{n-1} \frac{1-\cos \frac{2(n-1) k \pi}{n}}{2}\left[1-\sin \frac{2(n-1) k \pi}{n}\right] \\
&
\end{aligned}
$$




$$
\begin{aligned}
= & \frac{2}{n(n-4)} \sum_{k=1}^{n-1}\left[1-\sin \frac{2(n-1) k \pi}{n}-\cos \frac{2(n-1) k \pi}{n}+\right. \\
& \left.\frac{1}{2} \sin \frac{4(n-1) k \pi}{n}\right] \\
= & \frac{2}{n(n-4)}\left[n-1-\sum_{k=1}^{n-1} \sin \frac{2(n-1) k \pi}{n}-\sum_{k=1}^{n-1} \cos \frac{2(n-1) k \pi}{n}+\right. \\
& \left.\frac{1}{2} \sum_{k=1}^{n-1} \sin \frac{4(n-1) k \pi}{n}\right] \\
= & \frac{2}{n(n-4)}[n-1-0-(-1)+0]=\frac{2}{n-4},
\end{aligned}
$$

as required.

\section{APPENDIX B: PROOF OF PROPOSITION 3.3.}

We first introduce a result, which plays an essential role in the proof of Proposition 3.3.

Proposition 5.1. (The reduction principle) ${ }^{50}$ If $S \subset V(G)$ satisfies that all vertices in $S$ have the same neighborhood $N$ in $G-S$, then resistance distances between vertices in $S$ can be computed as in the subgraph obtained from $G[S \cup N]$ by deleting all the edges between vertices in $N$.

Now we are ready to prove Proposition 3.3.

Proof. First assume that $p, q \in V(G / S)$ and we consider resistance distance between $p$ and $q$ in $G$. If we apply a potential difference between $p$ and $q$, by symmetry we know that all the vertices in $S$ have the same potential and thus they could be shorten together. Noticing that $G / S$ will result if we short all the vertices of $S$ together, we readily have $\Omega_{G / S}(p, q)=\Omega_{G}(p, q)$.

Now assume that $p \in V(G) \backslash S$ and $q \in S$. We will prove Equation (3.4) by induction on the cardinality of $S$. Suppose, first, that $|S|=2$ and $S=\left\{q, v_{1}\right\}$. Then by symmetry, it is easily seen that $\Omega_{G}(p, q)=\Omega_{G}\left(p, v_{1}\right)$. Hence by Theorem 3.2, we have

$$
\begin{aligned}
& \Omega_{G / S}(p, q)=\Omega_{G}(p, q)- \\
& \frac{\left[\Omega_{G}(p, q)+\Omega_{G}\left(q, v_{1}\right)-\Omega_{G}\left(p, v_{1}\right)-\Omega_{G}(q, q)\right]^{2}}{4 \Omega_{G}\left(q, v_{1}\right)} \\
& =\Omega_{G}(p, q)-\frac{\Omega_{G}^{2}\left(q, v_{1}\right)}{4 \Omega_{G}\left(q, v_{1}\right)} \\
& =\Omega_{G}(p, q)-\frac{\Omega_{G}\left(q, v_{1}\right)}{4} .
\end{aligned}
$$

Then by the reduction principle (Proposition 5.1), the resistance distance between $q$ and $v_{1}$ in $G$ can be computed as in the subgraph obtained from $G[S \cup N]$ by deleting all the edges between vertices in $N$. Noticing that the subgraph is the complete bipartite graph $K_{2,|N|}$, it follows that ${ }^{32}$

$$
\Omega_{G}\left(q, v_{1}\right)=\Omega_{K_{2,|N|}}\left(q, v_{1}\right)=\frac{2}{|N|} .
$$

Substituting Equation (5.3) into Equation (5.2), we have

$\Omega_{G / S}(p, q)=\Omega_{G}(p, q)-\frac{\Omega_{G}\left(q, v_{1}\right)}{4}=\Omega_{G}(p, q)-\frac{1}{|N|}$,

as desired.

Suppose, now, that the assertion holds for $S$ whenever $|S| \leq k$, and consider the case that $|S|=k+1$. Suppose that $S=\left\{q, v_{1}, v_{2}, \ldots, v_{k}\right\}$. For $1 \leq i \leq k$, let $S_{i}=\left\{v_{1}, v_{2}, \ldots, v_{i}\right\}$. Firstly we show the following claim.

Claim.

$$
\Omega_{G / S_{k}}\left(q, v_{k}\right)=\frac{k+1}{|N| k}
$$

Proof of the Claim. Since $G / S_{k}$ could be obtained from $G / S_{k-1}$ by shorting $v_{k}$ and $v_{k-1}$ together, by Theorem 3.2, we have

$$
\begin{aligned}
& \Omega_{G / S_{k}}\left(q, v_{k}\right)=\Omega_{G / S_{k-1}}\left(q, v_{k}\right)- \\
& \frac{\left[\Omega_{G / S_{k-1}}\left(v_{k-1}, v_{k}\right)+\Omega_{G / S_{k-1}}\left(q, v_{k}\right)-\Omega_{G / S_{k-1}}\left(q, v_{k-1}\right)\right]^{2}}{4 \Omega_{G / S_{k-1}}\left(v_{k-1}, v_{k}\right)} .
\end{aligned}
$$

In $G$, if we attach an battery accross $v_{k}$ and $q$, then by symmetry, vertices $v_{1}, v_{2}, \ldots, v_{k-1}$ have the same potential and thus shorting them together does not change the effective resistance between $v_{k}$ and $q$. Therefore,

$$
\Omega_{G / S_{k-1}}\left(q, v_{k}\right)=\Omega_{G}\left(q, v_{k}\right)
$$

Noticing that the subgraph obtained from $G[S \cup N]$ by deleting all the edges between vertices in $N$ is the complete bipartite graph $K_{k+1,|N|}$, so again by the reduction principle, it is easy to compute that

$$
\Omega_{G}\left(q, v_{k}\right)=\Omega_{K_{k+1,|N|}}\left(q, v_{k}\right)=\frac{2}{|N|} .
$$

Hence resistance distance between any two vertices in $S$ is $\frac{2}{|N|}$ in $G$.

On the other hand, by the induction hypothesis, we have 


$$
\begin{aligned}
& \Omega_{G / S_{k-1}}\left(v_{k}, v_{k-1}\right)=\Omega_{G}\left(v_{k}, v_{k-1}\right)-\frac{\left|S_{k-1}\right|-1}{\left|S_{k-1}\right| N \mid} \\
& =\frac{2}{|N|}-\frac{k-2}{(k-1)|N|}=\frac{k}{|N|(k-1)}, \\
& \Omega_{G / S_{k-1}}\left(q, v_{k-1}\right)=\Omega_{G}\left(q, v_{k-1}\right)-\frac{\left|S_{k-1}\right|-1}{\left|S_{k-1}\right| N \mid} \\
& =\frac{2}{|N|}-\frac{k-2}{(k-1)|N|}=\frac{k}{|N|(k-1)} .
\end{aligned}
$$

Substituting Equations (5.6), (5.7), (5.8) and (5.9) back into Equation (5.5), simple calculation yields the claim.

Now we proceed to prove Equation (3.4). By Theorem 3.2 ,

$$
\begin{aligned}
& \Omega_{G / S}(p, q)=\Omega_{G / S_{k}}(p, q)- \\
& \frac{\left[\Omega_{G / S_{k}}(p, q)+\Omega_{G / S_{k}}\left(v_{k}, q\right)-\Omega_{G / S_{k}}\left(p, v_{k}\right)\right]^{2}}{4 \Omega_{G / S_{k}}\left(v_{k}, q\right)} .
\end{aligned}
$$

If we attach a battery across $p$ and $q$ in $G$, then vertices $v_{1}, v_{2}, \ldots, v_{k}$ have the same potential and they may be shorten together without affecting the resistance distance between $p$ and $q$. This implies that

$$
\Omega_{G / S_{k}}(p, q)=\Omega_{G}(p, q) .
$$

By Equation (5.4), we have

$$
\Omega_{G / S_{k}}\left(v_{k}, q\right)=\frac{k+1}{|N| k} .
$$

Noticing that $\Omega_{G}\left(p, v_{k}\right)=\Omega_{G}(p, q)$, by the induction hypothesis, we have

$$
\begin{aligned}
\Omega_{G / S_{k}}\left(p, v_{k}\right) & =\Omega_{G}\left(p, v_{k}\right)-\frac{k-1}{|N| k} \\
& =\Omega_{G}(p, q)-\frac{k-1}{|N| k} .
\end{aligned}
$$

Substituting Equations (5.11), (5.12) and (5.13) into Equation (5.10), we have

$$
\begin{aligned}
\Omega_{G / S}(p, q) & =\Omega_{G}(p, q)- \\
& \frac{\left[\Omega_{G}(p, q)+\frac{k+1}{|N| k}-\left(\Omega_{G}(p, q)-\frac{k-1}{|N| k}\right)\right]^{2}}{4 \frac{k+1}{|N| k}} \\
& =\Omega_{G}(p, q)-\frac{k}{|N|(k+1)}
\end{aligned}
$$

$$
=\Omega_{G}(p, q)-\frac{|S|-1}{|S||N|},
$$

and the proof is complete.

\section{REFERENCES}

1. D. J. Klein and M. Randić, J. Math. Chem. 12 (1993) 81-95.

2. G. Kirchhoff, Ann. Phys. Chem. 72 (1847) 497-508.

3. J. C. Maxwell, Electricity and Magnetism, Clarendon Press, Oxford, 1892.

4. S. Seshu and M. B. Reed, Linear Graphs and Electrical Networks, Addison-Wesley, Reading, Mass, 1961.

5. S. P. Chan, Introductory Topological Analysis of Electrical Networks, Holt, Rinehart and Winston, New York, 1969.

6. P. G. Doyle and J. L. Snell, Random Walks and Electric Networks, The Mathematical Association of America, Washington, DC, 1984.

7. B. van der Pol, The finite-difference analogy of the periodic wave equation and the potential equation, Appendix IV, in: M. Kac (Ed.), Probability and Related Topics in Physical Sciences, Interscience, London, 1959, pp. 237-257.

8. S. Katsura, T. Morita, S. Inawashiro, T. Horiguchi, and Y. Abe, J. Math. Phys. 12 (1971) 892-895.

9. H. Chen and F. Zhang, Discrete Appl. Math. 155 (2007) 654661.

10. I. Gutman, L. Feng, and L. Yu, Trans. Comb. 1 (2012) 27-40.

11. D. J. Klein, I. Lukovits, and I. Gutman, J. Chem. Inf. Comput. Sci. 35 (1995) 50-52.

12. H.-Y. Zhu, D. J. Klein, and I. Lukovits, J. Chem. Inf. Comput. Sci. 36 (1996) 420-428.

13. H.-Y. Zhu and D. J. Klein, J. Chem. Inf. Comput. Sci. 36 (1996) 1067-1075.

14. O. Ivanciuc, ACH-Models Chem. 137 (2000), 607-631.

15. O. Ivanciuc, Rev. Roum. Chim. 47 (2002) 675-686.

16. O. Ivanciuc and D. J. Klein, Croat. Chem. Acta 75 (2002) $577-$ 601.

17. O. Ivanciuc and D. J. Klein, J. Chem. Inf. Comput. Sci. 42 (2002) 8-22.

18. B. Zhou and N. Trinajstić, Croat. Chem. Acta 83 (2010) $227-$ 242.

19. C. St. J. A. Nash-Williams, Proc. Cambridge Phil. Soc. 55 (1959) 181-194.

20. D. J. Klein, MATCH Commun. Math. Comput. Chem. 35 (1997) 7-27.

21. J. L. Palacios, Int. J. Quantum Chem. 81 (2001) 29-33.

22. R. B. Bapat, I. Gutman, and W. Xiao, Z. Naturforsch. 58a (2003) 494-498.

23. W. J. Xiao and I. Gutman, Theor. Chem. Acc. 110 (2003) 284 289.

24. D. Bonchev, A. T. Balaban, X. Liu, and D. J. Klein, Int. J. Quantum Chem. 50 (1994) 1-20.

25. D. J. Klein and O. Ivanciuc, J. Math. Chem. 30 (2001) 271-287.

26. D. J. Klein, J. Math. Chem. 47 (2010) 1207-1233.

27. R. B. Bapat, Math. Student 68 (1999) 87-99.

28. I. Lukovits, S. Nikolić, and N. Trinajstić, Int. J. Quantum Chem. 71 (1999) 217-225.

29. I. Lukovits, S. Nikolić, and N. Trinajstić, Croat. Chem. Acta 73 (2000) 957-967.

30. A. T. Balaban and D. J. Klein, Scientometrics 55 (2002) 59-70.

31. D. J. Klein and H.-Y. Zhu, J. Math. Chem. 23 (1998) 179-195.

32. D. J. Klein, Croat. Chem. Acta 75 (2002) 633-649.

33. D. J. Klein, J. L. Palacios, M. Randić, and N. Trinajstić, J. Chem. Inf. Comput. Sci. 44 (2004) 1521-1525. 
34. O. Ivanciuc, T. Ivanciuc, and D. J. Klein, MATCH Commun Math. Comput. Chem. 44 (2001) 251-278.

35. P. W. Fowler, Croat. Chem. Acta 75 (2002) 401-408.

36. F. Y. Wu, J. Phys. A: Math. Gen. 37 (2004) 6653-6673.

37. M. A. Jafarizadeh, R. Sufiani, and S. Jafarizadeh, J. Phys. A. Math. Theor. 40 (2007) 4949-4972.

38. M. A. Jafarizadeh, R. Sufiani, and S. Jafarizadeh, J. Math. Phys. 49 (2008) 073303.

39. M. A. Jafarizadeh, R. Sufiani, and S. Jafarizadeh, J. Math. Phys. 50 (2009) 023302

40. S. Jafarizadeh, R. Sufiani, and M. A. Jafarizadeh, J. Stat. Phys. 139 (2010) 177-199.

41. H. Chen and F. Zhang, J. Math. Chem. 44 (2008) 405-417.

42. H. Chen, Discrete Appl. Math. 158 (2010) 1691-1700.
43. B. Zhou and N. Trinajstić, J. Math. Chem. 46 (2009) 283-289.

44. Y. Yang, Dig. J. Nanomater. Bios. 7 (2012) 593-598.

45. A. D. G. Maden, I. Gutman, and A. S. Çevic, Hacet. J. Math. Stat. 42 (2013) 43-50.

46. Y. Yang and D. J. Klein, Discrete Appl. Math. 161 (2013) 2702 2715

47. Q. Deng and H. Chen, Linear Algebra Appl. 439 (2013) $167-$ 173

48. H. Zhang and Y. Yang, Int. J. Quantum Chem. 107 (2007) 330339.

49. Y. Li and Y. Wang, J. Yantai Uni. (Nat. Sci.) 4 (2012) 235-238 (in Chinese)

50. Y. Yang and H. Zhang, J. Phys. A: Math. Theor. 41 (2008) 445203 . 